Cantarero Tomás, David.

Artista visual / Doctorando, Programa de Doctorado en Arte: Producción e Investigación, Facultad de Bellas Artes de San Carlos, Universitat Politècnica de València.

\title{
El uso no normativo de las nuevas tecnologías en la práctica artística contemporánea. Estudio de caso: obras Tecnofósil l y Tecnofósil II.
}

\section{New technologies creative misuse in Contemporary Art Practice. Case study: artworks Technofossil I \& Technofossil II.}

\author{
TIPO DE TRABAJO:
}

Póster.

PALABRAS CLAVE:

Antropoceno, estereoscopía, extrañamiento, impresión 3D, tecnofósil, uso no normativo.

KEY WORDS:

Anthropocene, creative misuse, defamiliarization, stereoscopy, technofossil, 3D printing.

RESUMEN.

La utilización de materiales, procesos y técnicas de un modo contrario a la norma y con una intención crítica, es algo que podemos encontrar en diversas manifestaciones artísticas ya desde comienzos del s. XX. Sin embargo, es con el surgimiento de nuevos aparatos electrónicos a mediados de siglo y su accesibilidad cada vez mayor, cómo este modo de hacer cobra una nueva dimensión y llega a convertirse en un recurso extendido dentro de la práctica artística contemporánea. En el siguiente trabajo se aborda este tema desde una perspectiva teórico-práctica, a través del análisis de una serie de obras de producción propia que, mediante el uso no normativo o estipulado como el correcto de diversas tecnologías, tratan de conectar y conducir la reflexión sobre varias problemáticas de alcance global referentes tanto a la imagen como al impacto de la técnica a diferentes niveles. Combinando libremente el empleo de herramientas como la impresión 3D o la estereoscopía, en torno a ciertos materiales extraídos de una de las playas cementadas de la costa vasca, enclave considerado como paradigmático a la hora de definir y estudiar la etapa geológica que ha venido a denominarse Antropoceno, en estas piezas se abordan cuestiones relativas a los modos de circulación con que cuenta la imagen hoy en día, así como a la actualidad de nociones como las de representación o mímesis, aspectos referentes a la incidencia de los medios de comunicación de masas y de la tecnología tanto a nivel social como individual, o ideas vinculadas a la alteración y modificación de multiplicidad de procesos naturales como consecuencia de la actividad humana.

\section{ABSTRACT.}

The use of materials, processes and techniques in a way contrary to the norm and with a critical intention, is something that we can find in various artistic manifestations since the beginning of the twentieth century. However, it is with the emergence of new electronic devices in the middle of the century and their increasing accessibility, how this way of doing takes on a new dimension and becomes an extended resource within the contemporary art practice. In the following work this subject is approached from a theoretical-practical perspective, through the analysis of a series of works of own production that, by the non-normative use or stipulated as the correct one of diverse technologies, try to connect and conduct the reflection on several issues of global scope concerning both the image and the impact of the technique at different levels. Combining freely the use of tools such as 3D printing or stereoscopy, around certain materials extracted 
from one of the cemented beaches of the Basque coast, an enclave considered as paradigmatic in defining and studying the geological stage that has come to be called Anthropocene, these pieces deal with issues related to the modes of circulation of the image nowadays, as well as to the current validity of notions such as representation or mimesis, aspects related to the incidence of the mass media and of technology both socially and individually, or ideas linked to the alteration and modification of multiplicity of natural processes as a consequence of human activity.

\section{CONTENIDO.}

\section{INTRODUCCIÓN.}

El uso de procedimientos, aparatos y dispositivos tecnológicos de diversa índole de un modo diferente a aquel para el cual habían sido pensados o expresamente creados, es una constante en la práctica artística de las últimas décadas. Podemos seguir el impulso inicial de este comportamiento hasta algunos de los movimientos de vanguardia de principios del siglo XX como el Dadaísmo, con obras como los ready-made de Duchamp, o ya entrados en la segunda mitad de siglo, encontrarlo en movimientos como el Situacionismo con estrategias como el détournement, o el Fluxus con la introducción deliberada del azar y de lo imprevisto en las obras, como en las partituras para piano preparado que popularizó Jonh Cage. Dentro de este mismo movimiento, y vinculada ya a la llegada de multiplicidad de aparatos electrónicos y su accesibilidad cada vez mayor, podemos destacar la figura de Nam June Paik, cuya obra procede constantemente a través de ese "mal uso" deliberado y creativo de la tecnología, ${ }^{1}$ pudiendo ver en este artista uno de los precursores de muchas de las prácticas que vendrán después con la aparición de internet y las nuevas tecnologías.

Es posible así reconocer hoy esa misma intención crítica, el deseo de cambiar la realidad social y política en la se encuentran, en nuevas expresiones que irían desde prácticas como el glitch art o el circuit bending, a manifestaciones que en muchas ocasiones exceden la exclusiva esfera del arte, como el hacking o el culture jamming. En todas ellas se opera mediante el uso no ortodoxo, subversivo y distorsionado de herramientas, técnicas y lenguajes, con la intención de generar interferencias, reestructurar, rediseñar, reinventar, haciendo en cada caso énfasis en el proceso, los dispositivos o los resultados obtenidos a través de ellos, dependiendo de los objetivos que se persigan.
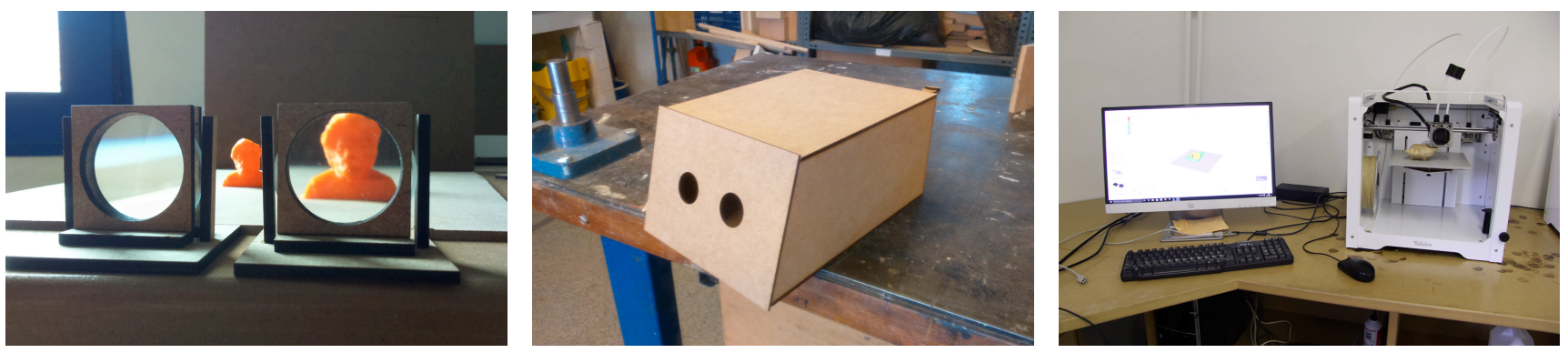

Figura 1. Imágenes del proceso de producción de las obras; a) primeras pruebas para ajustar las dimensiones de la caja, así como la de los elementos a colocar en el interior de la misma, en relación a la distancia focal y el tamaño de las lentes; b) montaje de una de las cajas tras obtener las diferentes piezas que la componen mediante corte láser; c) equipo de impresión 3D con el que se han realizado los modelos en termoplástico ABS.

\section{TECNOFÓSIL I Y II (GORRONDATXE).}

\section{A. Estereoscopía tridimensional.}

Las dos obras que se analizan en este trabajo, pertenecientes a una misma serie, ponen en funcionamiento lo que de forma crítica y un tanto irónica he denominado "estereoscopía tridimensional". En el interior de cada una de estas cajas (fig. 1), se ha dispuesto una piedra y una reproducción de la misma impresa en 3D a escala real, previa obtención del modelo mediante escaneo 3D (fig. 2), de tal manera que cada uno de estos elementos es visible únicamente para cada uno de los dos ojos. Al ser observados a través de unas

\footnotetext{
${ }^{1}$ Un breve pero interesante análisis de este tipo de prácticas a lo largo del s. XX, haciendo especial hincapié en el mencionado artista, puede encontrarse en Jon Ippolito, The Art of Misuse.
} 
lentes similares a las utilizadas en las actuales gafas de 3D diseñadas para dispositivos móviles, obtenemos una imagen resultante que es fruto de la superposición de las dos vistas independientes (fig. 5). Es sin embargo una imagen que presenta ciertas distorsiones y desajustes debido a las diferencias entre ambos elementos, una suerte de mezcla entre objeto real y reproducción que no acaba de consumarse, que es problemática, que perturba, que incomoda.
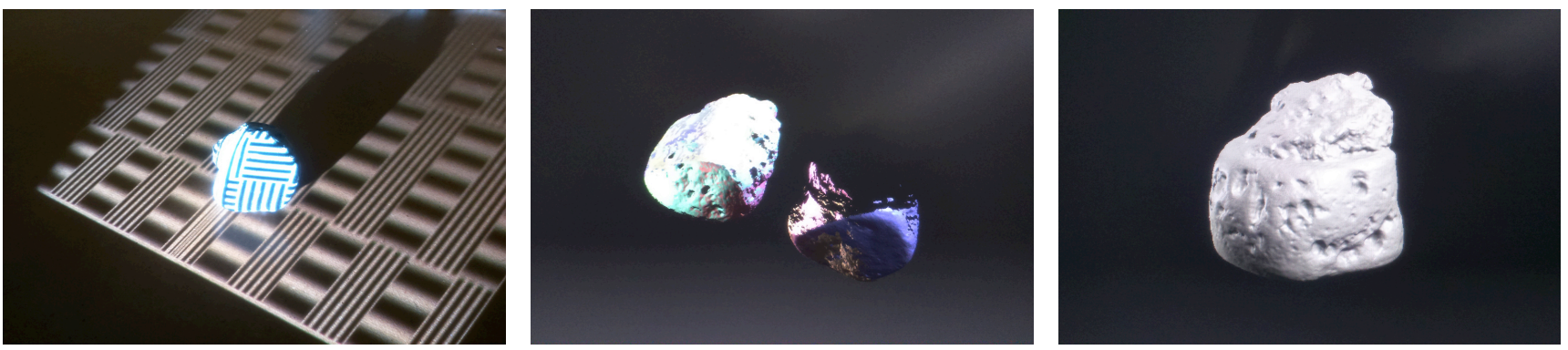

Figura 2. Imágenes del proceso de producción de las obras; a) proceso de escaneado 3D de una de las piedras extraídas de la playa de Gorrondatxe; b) generación del modelo 3D en el que se procede ensamblando las diferentes vistas obtenidas del objeto; c) vista del modelo 3D finalizado, listo para ser exportado a un archivo para impresión 3D.

\section{B. Antropoceno.}

No obstante, esos objetos colocados en el interior de las cajas no son, pese a parecerlo, simples piedras, sino lo que han venido a denominarse "tecnofósiles". ${ }^{2}$ Se ha superpuesto de este modo, casi replicando el efecto dado a nivel visual, una nueva capa de lectura, ya que a partir de esos pequeños fragmentos obtenidos de un paraje particular de la costa vasca (fig. 3), la playa cementada de Gorrondatxe, ${ }^{3}$ se trata de reflexionar sobre el impacto de la actividad humana en multiplicidad de procesos geológicos y biológicos, a escala planetaria. Los sedimentos presentes en esta y otras playas anexas, originados por decenas de años de vertidos al mar de la escoria y los residuos provenientes principalmente de los Altos Hornos de Vizcaya, ${ }^{4}$ son la parte visible de un proceso de alcance global que ha llevado a proponer un nuevo término, el de "Antropoceno", 5 para dar cuenta de un cambio de era. ${ }^{6}$ Este enclave se ha convertido -junto con otros ampliamente conocidos, como la playa hawaiana de "plastiglomerados" ${ }^{7}$ Kamilo Beach- en un ejemplo destacado dentro del ámbito de la investigación geológica, a la hora de definir y acotar temporalmente este fragmento de la historia de la Tierra en el que el impacto de la actividad antrópica vinculada a la industrialización se ha hecho evidente. ${ }^{8}$
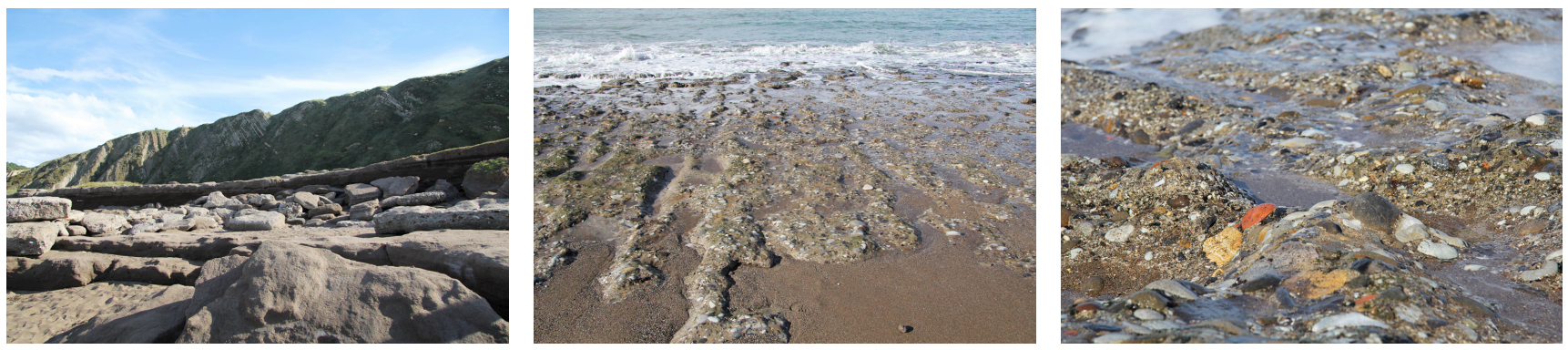

Figura 3. Playa cementada de Gorrondatxe, Bizkaia, País Vasco; a) vista general en la que se puede apreciar el desnivel existente entre los dos grupos de estratos que componen la playa principalmente; b) fotografía de los depósitos conglomeráticos cementados del estrato inferior; c) detalle en el que se pueden observar los diferentes tipos de escorias que conforman los depósitos, entre ellos fragmentos de ladrillo refractario.

\footnotetext{
${ }^{2}$ Aunque han sido modificados por la acción de diferentes procesos naturales, su origen es industrial, pudiendo ser incluidos en esta categoría de reciente cuño: Jan Zalasiewicz et al., "The technofossil record of humans", citado en Victoriano Pujalte et al., "Las playas cementadas del «Antropoceno» de Bizkaia", 126.

${ }^{3}$ Esta parte del litoral está dentro de la lista de "Lugares de interés geológico de la Comunidad Autónoma del País Vasco". Puede encontrase información más detallada en la web del Departamento de Medio Ambiente, Planificación Territorial y Vivienda del Gobierno Vasco.

${ }^{4}$ Victoriano Pujalte, op. cit.

${ }^{5}$ Paul J. Crutzen y Eugene F. Stoermer, "The «Anthropocene»", citado en Pujalte, op. cit., 123.

${ }^{6}$ La noción de "Antropoceno" no cuenta con consenso dentro de la comunidad científica internacional y está pendiente a día de hoy de tener reconocimiento oficial. Puede profundizarse en este tema en: Colin N. Waters et al., "The Anthropocene is functionally and stratigraphically distinct from the Holocene", citado en Kirsty Robertson, "Plastiglomerate", 5.

${ }^{7}$ Un texto en el que se aborda desde la perspectiva del arte la relación existente entre este "nuevo material" y diferentes aspectos como la contaminación, el impacto de la actividad humana en los ecosistemas a escala planetaria y la visibilidad, es el citado artículo de Kirsty Robertson "Plastiglomerate".

${ }^{8}$ Pujalte, op. cit., 126.
} 

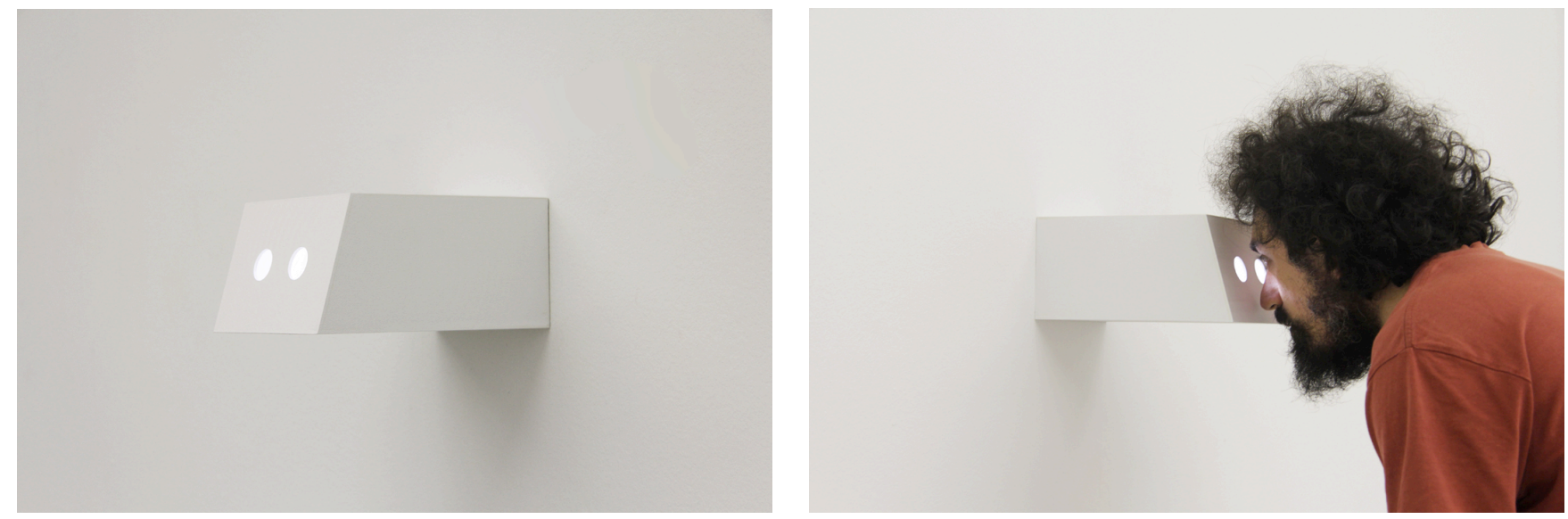

Figura 4. Fotografías de una de las piezas instalada; Tecnofósil (Gorrondatxe), 2016. Estereoscopía tridimensional: caja (DM), lentes biconvexas, placa LED, piedra, impresión 3D (ABS). $12 \times 18 \times 25 \mathrm{~cm}$.

\section{CONCLUSIONES.}

En estas obras se pretende generar cierto efecto de extrañamiento ${ }^{9}$ a nivel visual fruto de la superposición de los objetos y sus propias reproducciones, en un juego de ocultación-mostración-saturación. ${ }^{10}$ Con la invención de un dispositivo de fabricación propia que mezcla la elaboración artesanal con el uso de tecnologías de reciente aparición, ${ }^{11}$ la intención es tratar de ofrecer una mirada crítica sobre la expansión y uso de multiplicidad de dispositivos de visión estereoscópica y 3D -como los de realidad aumentada y virtual-, trastocando, interrumpiendo y distorsionando el normal funcionamiento de estos mecanismos. Se trata de crear a nivel simbólico una fisura visible y en lo visible, propiciar una distancia dentro de lo que Baudrillard ha calificado de "realidad integral", ${ }^{12}$ interfiriendo, siguiendo a Rancière, en los flujos de distribución de lo visible ${ }^{13}$ de lo que autores como Brea han denominado un nuevo régimen escópico de "hipervisión administrada". ${ }^{14}$

Si lo que tratan las mencionadas tecnologías es de dar un modelo lo más fiel posible, de disimular la diferencia, de evitar la falla, aquí lo que se pretende es evidenciar el error, prestar atención al desajuste, a los detalles que al final son lo más propio del proceso. Ya que al igual que en los que se dan a pequeña escala, en los grandes y masivos, lo que escapa, la falla, lo imprevisto, tal vez es la clave para un entendimiento mayor y más complejo. Retomando un último pensamiento, estos fósiles de la época industrial, al igual que los plastiglomerados sobre los que reflexionaba Kirsty Robertson en su artículo, son "un resto, un recordatorio, un indicador de la lenta violencia de la polución masiva". ${ }^{15}$

\footnotetext{
${ }^{9}$ El uso del extrañamiento como recurso en el arte fue teorizado por primera vez en 1917 por Viktor Shklovsky en su ensayo "El arte como técnica". Originalmente planteado como "ostranenie" y estrechamente vinculado al formalismo y la literatura rusa, se ha relacionado posteriormente con varias disciplinas artísticas, cambiando su significado e implicaciones en parte como resultado de las diferentes traducciones sufridas - por ejemplo, la incorporación por parte de Bertolt Brecht al teatro como "distanciamiento", o su traducción dentro del contexto anglosajón como "defamiliarization" (desfamiliarización). Dentro de las artes visuales podríamos vincularlo a diversos procedimientos y modos de hacer, cuestión sobre la que actualmente me encuentro investigado dentro de mi proyecto de tesis doctoral. ${ }^{0}$ Estos mecanismos, relacionados con la mencionada estrategia del extrañamiento, son los que se han ido utilizando a través de diferentes procedimientos dentro de la línea de investigación a la que pertenecen estas piezas. Estas obras en particular han sido producidas en el trascurso de una beca de colaboración realizada en la Fundación BilbaoArte durante el 2016 . Puede encontrarse información más detallada en mi página web http://www.davidcantarerotomas.com/ y en mi blog http://dacanto.blogspot.com.es/ ${ }^{11}$ Principalmente el escaneo y la impresión 3D en sus versiones más recientes, ya que la invención de las primeras técnicas de impresión $3 \mathrm{D}$ se remonta a los años 80 , y diferentes aparatos de visión estereoscópica, como por ejemplo el estereoscopio de Holmes, datan de mediados del s. XIX.

12 Jean Baudrillard, La agonía del poder, 60.

13 Jacques Rancière, "El teatro de las imágenes", en Alfredo Jaar et al., La Política de las imágenes.

14 José Luís Brea, Las tres eras de la imagen, 122.

${ }^{15}$ Kirsty Robertson, op. cit., 10.
} 
El uso no normativo de las nuevas tecnologías en la práctica artística contemporánea. Estudio de caso: obras Tecnofósil I y Tecnofósil II

III CONGRESO INTERNACIONAL DE INVESTIGACIÓN EN ARTES VISUALES :: ANIAV 2017 :: GLOCAL [codificar, mediar, transformar, vivir] http://dx.doi.org/10.4995/ANIAV.2017.4835
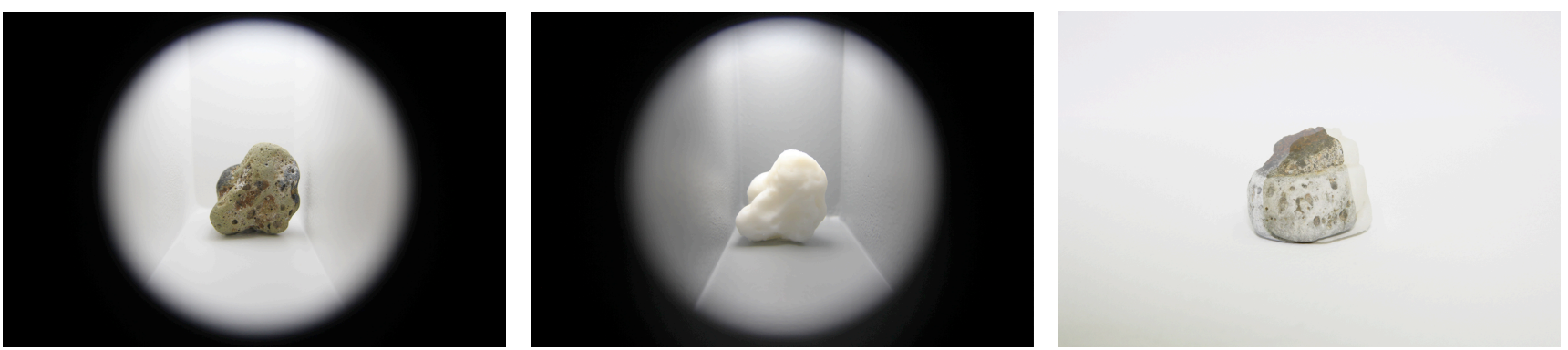

Figura 5. a) y b) fotografías pertenecientes a una de las obras finalizada, en este caso Tecnofósil II. Cada una de las imágenes corresponde a la vista que se obtiene por cada uno de los dos ojos, en el mismo orden en el que están dispuestas: el objeto real en el lado izquierdo y el modelo en 3D en el derecho; c) simulación de lo sería la imagen resultante, al fundirse-superponerse las vistas de cada ojo, en este caso de Tecnofósil I.

\section{FUENTES REFERENCIALES.}

Baudrillard, Jean, La agonía del poder, Madrid, Círculo de Bellas Artes, 2006.

Brea, José Luís, Las tres eras de la imagen. Imagen-materia, film, e-image, Madrid, Akal, 2010.

Crutzen, Paul J., y Stoermer, Eugene F., "The "Anthropocene»", Global Change Newsletter 41 (2000): 17-18 [consulta: 10-03-2017]. Disponible en: http://www.igbp.net/download/18.316f18321323470177580001401/1376383088452/NL41.pdf

Departamento de Medio Ambiente, Planificación Territorial y Vivienda, Gobierno Vasco, "Playa cementada de Gorrondatxe y Tunelboca - Lugares de interés geológico de la Comunidad Autónoma del País Vasco" [consulta: 10-03-2017]. Disponible en: http://www.ingurumena.ejgv.euskadi.eus/r49-

u95/es/u95aWar/lugaresJSP/U95aEConsultaLugar.do?u95aMigasPan=L,7,1;EN,0,14,0,300;\&pk

Ippolito, Jon, "The Art of Misuse", en Telematic Connections: The Virtual Embrace, 2001 [consulta: 10-03-2017]. Disponible en: http://telematic.walkerart.org/overview/overview_ippolito.html

Pujalte, Victoriano, Astibia, Humberto, Aizpiri, Fernando, y Payros, Aitor, "Las playas cementadas del «Antropoceno» de Bizkaia, País Vasco: origen y degradación”, Geogaceta 57 (2015): 123-126 [consulta: 2017-03-10]. Disponible en: http://www.sociedadgeologica.es/archivos/geogacetas/geo57/G57art31.pdf

Rancière, Jacques, "El teatro de las imágenes", en Jaar, Alfredo, Didi-Huberman, Georges, Pollock, Griselda, Rancière, Jacques, Schweizer, Nicole, La Política de las imágenes, 74-75, Santiago de Chile, Metales Pesados, 2008.

Robertson, Kirsty, "Plastiglomerate", e-flux journal 78 (2016) [consulta: 2017-03-10]. Disponible en: http://www.eflux.com/journal/78/82878/plastiglomerate/

Waters, Colin N., et al., "The Anthropocene is functionally and stratigraphically distinct from the Holocene", Science, vol. 351 , no. 6269 (2016) [consulta: 2017-03-10]. Disponible en: https://doi.org/10.1126/science.aad2622

Zalasiewicz, Jan, Williams, Mark, Waters, Colin N., Barnosky, Anthony D., y Haff, Peter, "The technofossil record of humans", The Anthropocene Review 1(2014): 34-43 [consulta: 10-03-2017]. Disponible en: https://doi.org/10.1177/2053019613514953 